\title{
Salud intercultural: elementos para la construcción de sus bases conceptuales
}

\author{
Ana M Alarcón $\mathbf{M}^{\mathrm{la}}$, Aldo Vidal $\mathrm{H}^{2 b}$, \\ Jaime Neira Rozas ${ }^{3 c}$. \\ of intercultural health
}

Over the past few years, intercultural health has become an emerging issue in health policy. Intercultural health is an approach to create a better communication between patients and providers. In the short term, this approach incorporates patient's culture background in health care, improving intercultural communication strategies to generate, in the long term, a health system adapted to the medical culture of patients. This article explores the underlying concepts in the intercultural health approach, such as cultural diversity and medical systems as complex models of thoughts and behaviors (Rev Méd Chile 2003; 131: 1061-65).

(Key Words: Cultural characteristics; Cultural diversity; Health care evaluation mechanisms)

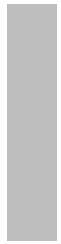 La Frontera y ${ }^{3}$ Servicio Salud Araucanía Norte.
aEnfermera, PhD Antropología Médica
bAntropólogo
'Magíster en Medicina Social

Recibido el 23 de julio, 2002. Aceptado en versión corregida el 19 de junio, 2003.

${ }_{1}^{1}$ Departamento de Salud Pública, Facultad de Medicina, Universidad de La Frontera. ${ }^{2}$ Departamento de Ciencias Sociales, Facultad de Educación y Humanidades, Universidad de

$\mathrm{E}^{\mathrm{n}}$ las últimas décadas, interculturalidad en salud o salud intercultural, han sido conceptos indistintamente utilizados para designar al conjunto de acciones y políticas que tienden a conocer e incorporar la cultura del usuario en el proceso de atención de salud. El tema de la pertinencia cultural del proceso de atención es

Correspondencia a: Ana M Alarcón M. Departamento de Salud Pública, Facultad de Medicina, Universidad de La Frontera. Montt 112, Temuco. Fono: 45-325738. Fax: 45-325777.

E mail: amalarc@ufro.cl un fenómeno que trasciende lo exclusivamente étnico pues implica valorar la diversidad biológica, cultural y social del ser humano como un factor importante en todo proceso de salud y enfermedad. El respeto a esta diversidad tiene larga trayectoria en países cuyas altas tasas de inmigración han generado contextos de gran diversidad étnica y cultural; situación que ha puesto al equipo de salud en el imperativo de desarrollar estrategias que faciliten la relación médico-paciente.

La necesidad de desarrollar procesos interculturales en salud se ha generado tanto en Chile como en otros países latinoamericanos por diver- 
sas razones históricas, sociopolíticas y epidemiológicas, las cuales han motivado iniciativas tendientes a evitar que la identidad étnica y cultural del usuario constituya una barrera en el acceso y oportunidad a una mejor atención de salud.

En este contexto, la antropología como ciencia contribuye tanto a develar y comprender las culturas involucradas en el proceso de atención de salud, como a proporcionar elementos socioantropológicos para apreciar el fenómeno de salud y enfermedad desde su dimensión biológica, social y cultural en un escenario de creciente pluralismo médico que caracteriza a la sociedad contemporánea.

Este artículo expone, desde la antropología médica, los conceptos fundamentales para el desarollo de un enfoque intercultural en salud, entre éstos, el concepto de cultura y diversidad cultural que subyacen en el estudio de los modelos médicos en interacción, y analiza los sistemas médicos como complejas y dinámicas organizaciones que representan modelos epistemológicos de pensamiento acerca del fenómeno de salud y enfermedad. En segundo lugar se analiza la interculturalidad en salud como un proceso de acercamiento entre mundos culturales diversos y se plantean algunas estrategias que permitinían implementar este enfoque en el sector salud.

\section{Cultura Y DIVERSIDAD CULTURAL EN SALUD}

El concepto de cultura aplicado al campo de la salud constituye el eje neurálgico en el análisis de los factores sociales involucrados en cualquier proceso de salud y enfermedad. A través del reconocimiento de las diferencias y similitudes culturales entre usuarios y proveedores es posible comprender, por ejemplo, el nivel y carácter de las expectativas que cada cual tiene del proceso, las percepciones de salud y enfermedad representadas en la interacción médico-paciente, los patrones con que se evalúa la calidad y resultado de la acción terapéutica, y el despliegue de diversas conductas frente a la enfermedad.

Desde la antropología, cultura se define como un complejo y dinámico conjunto de creencias, conocimientos, valores y conductas aprendidas y transmitidas entre las personas a través del lenguaje y su vida en sociedad. La cultura se entiende como una entidad dinámica que se adquiere, transforma y reproduce a través de un continuo proceso de aprendizaje y socialización ${ }^{1}$.

En el concepto de cultura convergen dos grandes dimensiones: una correspondiente a la ideacional o cognitiva que sintetiza el mundo simbólico de las personas, sus pensamientos, conocimientos y creencias adquiridas a través del aprendizaje, lo que podría llamarse el mundo de la conceptualización o de lo abstracto; y una dimensión material, que corresponde a cómo lo simbólico se manifiesta en conductas, prácticas, artefactos culturales y normas de relación social entre las personas 2,3 .

En el contexto de salud, estas dos dimensiones de la cultura, la cognitiva y material se articulan en un modelo médico que otorga consistencia a cómo pacientes y profesionales se explican el fenómeno de salud y enfermedad, y a las prácticas o procedimientos con que se enfrentan los procesos de recuperación y mantención de la salud. Normalmente los elementos cognitivos y prácticos difieren entre profesionales y pacientes producto de las diversas historias sociales y culturales de cada uno, del contexto en que ocurre el proceso de socialización de la cultura médica y del contenido propio de la cultura.

Las diferencias culturales entre pacientes y profesionales se observan también al interior de los grupos, lo cual refleja el carácter dinámico de la cultura. Como toda construcción humana los componentes cognitivos y materiales se transforman en la medida que el grupo social experimenta nuevos desafíos y problemas. Por ejemplo, tanto la biomedicina como las culturas médicas tradicionales experimentan transformaciones en sus modelos explicativos de enfermedad, incorporan nuevas tecnologías al proceso terapéutico y recrean roles profesionales, técnicos o empíricos para abordar aspectos específicos de salud de los usuarios.

\section{SISTEMAS MÉDICOS COMO SISTEMAS CULTURALES}

La enfermedad, sufrimiento y muerte, así como la motivación por conservar la salud, son considerados hechos biológicos y sociales universales. Todos los grupos humanos han desarrollado estrategias para enfrentar y prevenir las enfermedades a través de un sistema médico que proporciona las acciones nece- 
sarias para recuperar la salud, procurar el bienestar del hombre $\mathrm{y}$, al mismo tiempo, otorgar una explicación consistente al fenómeno de la enfermedad. Desde la antropología sociocultural un sistema médico se concibe como un conjunto más o menos organizado, coherente y estratificado de agentes terapéuticos, modelos explicativos de salud-enfermedad, prácticas y tecnologías al servicio de la salud individual y colectiva. La forma en que estos elementos se organizan internamente, otorgando coherencia al sistema depende del modelo sociocultural en que se desarrolla la medicina. En consecuencia las medicinas son construcciones culturales que responden a necesidades de un entorno social específico y en los cuales es posible distinguir una dimensión conceptual y otra conductual.

La dimensión conceptual de los sistemas médicos está determinada directamente por la cultura de los pacientes y profesionales. Analíticamente corresponde a los modelos que explican y fundamentan la enfermedad. O'Connor (1995; 1-34), sugiere que esta dimensión está compuesta por dos elementos estructurales: axiomas y mecanismos de validación*.

Todos los sistemas médicos presentan axiomas o principios basados en el modelo epistemológico que sustenta la práctica médica y distinguen medios para validarse y legitimarse a sí mismos. En la biomedicina por ejemplo, los experimentos científicos, las pruebas clínicas y textos, constituyen importantes fuentes de validación del pensamiento científico; raramente un médico aceptará como prueba de una diarrea la intervención de un espíritu maligno en las comidas. Sin embargo, otras culturas aceptarán como fuente de legitimación los sueños de un chamán, signos en la naturaleza, apariciones, etc. La lógica que opera en la definición de salud y enfermedad es la misma en ambos sistemas (una lógica que busca causas, alternativas y consecuencias), sin embargo difieren en las premisas culturales y pruebas de validación; por ello distintas percepciones y observaciones del mismo fenómeno resultan en diferentes explicaciones de la enfermedad.
La credibilidad que los pacientes asignan a las fuentes de validación es la que permite, en último término, aceptar o no las explicaciones acerca de las causas de sus enfermedades, y la adherencia a los tratamientos. Por lo tanto, el éxito en el proceso terapéutico depende en gran medida de la conjunción de explicaciones entre médicos y pacientes.

En la dimensión conductual de los sistemas de salud podemos distinguir, entre otros, aquellos procedimientos, acciones y agentes que utiliza un sistema médico para obtener un cierto resultado con los pacientes, el que ha sido denominado Proceso Terapéutico ${ }^{4-6}$. Este implica: quién (machi, médico, meica, santiguadora, etc.), hace qué (cirugía, ritual, purgas, oraciones, etc.), a quién (individuo o comunidad) y con respecto a qué dimensión de la enfermedad (física, social, mental, espiritual, etc.). Un proceso terapéutico exitoso supone la eliminación de la condición subjetiva de enfermedad y consecuentemente la finalización del rol de enfermo ${ }^{7}$.

\section{INTERCULTURALIDAD COMO PROCESO DE ACERCAMIENTO ENTRE SISTEMAS MÉDICOS}

En general, todos los procesos de interacción social y cultural que involucren la existencia de diferentes $\mathrm{y}$, a veces, antagónicos sistemas de creencias están sujetos a importantes fricciones. Sin embargo, los conflictos entre diferentes sistemas médicos no emergen sólo de las diferencias en los modelos explicativos que los sustentan, sino también de la dominación social de un modelo de salud sobre otro ${ }^{8,9}$. En este contexto la cultura biomédica expresada a través del sistema médico occidental, se ha establecido mundialmente como el modelo capaz de resolver si no todos, la mayoría de los problemas de salud de la población independientemente de los contextos sociales y culturales en que se desarrolle la enfermedad. No obstante, la experiencia de proyectos en salud internacional, muchos de ellos

\footnotetext{
* Axiomas: principios y definiciones fundamentales que no requieren pruebas para demostrar su existencia y resistentes a cualquier argumentación. Mecanismos de validación: hechos y evidencias que constatan el fenómeno observado o la creencia de los sujetos. Las evidencias son múltiples y diversas, desde pruebas de laboratorio hasta revelaciones chamánicas.
} 
patrocinados por organismos como la OPS y OMS, y dirigidos hacia los sectores más pobres de la humanidad, dan cuenta de importantes dificultades que se enfrentan al no considerar la cultura de los usuarios en el proceso de atención de salud. Situaciones como rechazo y falta de adhesión a las prácticas médicas occidentales, conflictos de poder entre agentes tradicionales y médicos, desarticulación de la estructura social de la comunidad, son elementos frecuentemente descritos en la literatura de salud internacional, especialmente en países con altos índices de población indígena.

Así, las variables étnico culturales y el respeto a la diversidad en la implementación de políticas sociales están pesando fuertemente en las propuestas de desarrollo de los países emergentes. En este contexto, el desafío para el sector salud en las próximas décadas será crear modelos de salud que respondan a las expectativas materiales y culturales del usuario, en un proceso de diálogo e intercambio cultural entre medicinas. En consecuencia, la búsqueda de estrategias que articulen e integren distintos enfoques de salud, es parte del escenario que hoy se nos presenta en el país en el sector salud, proceso que próximamente debutará con la generación de una política de salud intercultural, que transformará cualitativamente nuestro sistema haciéndolo más flexible y permeable a la realidad cultural del usuario.

\section{ESTRATEGIAS DE SALUD INTERCULTURAL}

Una política o enfoque intercultural en salud tiene al menos dos niveles de expresión: uno el de las acciones y actividades que materializan la interacción entre medicinas, y otro el de la cultura que sustenta los modelos médicos en interacción. Este último, el más complejo de desarrollar implica trascender desde la acción médica concreta hacia la implementación de un modelo pluricultural de salud y sociedad. Es un proceso que requiere transformaciones estructurales de largo plazo y responsabilidades que sobrepasan a los actores del sistema de salud, pues son las estructuras del Estado en conjunto con las sociedades indígenas quienes deben establecer las bases para un proyecto multicultural de sociedad.

A nivel de las acciones en salud, las estrategias de comunicación intercultural aparecen como factores que disminuyen las barreras sociales y culturales entre equipos de salud y usuarios. El mejorar los procesos de comunicación y diálogo, promueve una mayor comprensión de las expectativas de ambos actores y a la vez mejora el nivel de satisfacción de los usuarios con el sistema de salud biomédico. Al respecto, Berlin y Fowkes ${ }^{10}$ señalan que el éxito de la comunicación intercultural puede ser logrado a través de un proceso compuesto de cinco elementos: "escuchar, explicar, reconocer, recomendar y negociar". Los autores reconocen que aunque el lenguaje puede ser una gran barrera en la comunicación en sus aspectos gramaticales, simbólicos y semánticos, él no constituye un obstáculo infranqueable en la relación profesionalpaciente, si los profesionales están abiertos al diálogo y conocen en parte la cultura médica de sus pacientes, como por ejemplo, las denominaciones de enfermedad, la relación entre hábitos y cultura, la dimensión corporal del sufrimiento y la utilización de herbolaria tradicional, entre otras.

Una segunda estrategia de salud intercultural se refiere al desarrollo de procesos de validación y negociación cultural entre pacientes y profesionales. La validación cultural consiste en aceptar la legitimidad del modelo de salud y enfermedad del paciente considerando el contexto cultural en que este modelo emerge. En otras palabras las acciones de los pacientes frente a su enfermedad son la mayoría de las veces congruentes con las explicaciones aprendidas en su grupo social y cultural. La validación cultural no significa que el profesional comparta el mundo simbólico del paciente, sino que comprenda, respete e incluso integre algunos elementos culturales que considere relevantes para el proceso de recuperación del enfermo.

La negociación cultural se manifiesta cuando las creencias culturales y las expectativas de los pacientes se contraponen a las del profesional de salud interfiniendo en la confianza y adherencia al proceso terapéutico. La investigación médico etnográfica da cuenta, por ejemplo, de la escasa adherencia a tratamientos farmacológicos cuando son considerados nocivos por el paciente o en oposición a su marco de creencias ${ }^{11,12}$. El rechazo cultural hacia el procedimiento de donación de órganos y trasplantes documentado en Chile entre población mapuche, es otro ejemplo de cómo la cosmovisión sobre la vida y la muerte interfiere en una determinación médica tan importante como preservar la vida a través de una intervención quinúrgica ${ }^{13}$. Situaciones de esta índole 
son ampliamente documentadas en la literatura médico antropológica y demuestran que en la relación médico-paciente existe la probabilidad de antagonismos entre los marcos conceptuales y valónicos de ambos actores del proceso terapéutico. En estos casos, el proceso de negociación cultural identifica las áreas de conflicto y acuerdo, localiza núcleos de significación entre ambas culturas que puedan implicar puntos de consenso y culmina con un acuerdo de cambio y cooperación entre pacientes y médicos. Al respecto Kleinman ${ }^{7}$ sostiene que la única forma de lograr un acto terapéutico eficaz es a través del compromiso entre el enfermo y el profesional, el que se logra sólo si la propuesta de recuperación se inserta en la vida simbólica y cultural del enfermo, y si la intervención es aceptable, significativa y satisfactonia tanto para médicos como pacientes.

\section{CONCLUSIONES}

El desarrollo de una política de salud intercultural es un tema reciente en el quehacer nacional. Por lo tanto, el diseño de estrategias que faciliten el mayor

\section{REFERENCIAS}

1. PARK A. Introducing Anthropology. An Integrated Approach. Mayfield Publishing Company. California. 2000.

2. HANDEWERKER WP. Quick Ethnography. Altamira Press. California. 2000.

3. GeERz C. Thick description: Toward an Interpretative Theory of Culture. En: Bohannan P, Glazer M, editores. High Points in Anthropology. McGraw-Hill. New York. 1998; 529-51.

4. O'Connor BB. Healing Traditions. Alternative Medicine and the Health Professions. University of Pennsylvania Press. 1995.

5. Kieinman A, Csordas T. The Therapeutic Process. En: Sargent C, Johnson T, editores. Medical Anthropology. Contemporary Theory and Method. Praeger Publishers. Wesport, Connecticut. 1996; 3-21.

6. HanHn RA. Sickness and Healing: An Anthropological Perspective. New Haven. Yale University Press. 1995.

7. Kizinman A. Writing at the margin. Discourse between Anthropology and Medicine. University diálogo y comunicación entre modelos médicos se encuentra abierto a la creatividad de quienes van a ejecutar las acciones en salud intercultural.

No obstante, la experiencia intemacional ha demostrado la existencia de principios conceptuales que sustentan el desarrollo de modelos pluriculturales en salud. Entre estos se encuentra el de reconocer a los sistemas médicos como complejos modelos de pensamiento y conducta, los cuales constituyen respuestas culturales al fenómeno universal de la enfermedad. Un segundo principio presentado en este artículo, es el de mejorar los procesos de comunicación entre pacientes y profesionales, fenómeno que no sólo involucra al lenguaje como acto comunicativo sino también al conocimiento de los protocolos de relación cultural y social de los pacientes.

En un contexto de salud intercultural, ambos ejes conceptuales, no debieran parecer ajenos o extraños a la práctica médica, ni tampoco implicar una carga para el profesional de salud dado que se fundamentan en los actuales principios de bioética médica expresado en el respeto a la autonomía de los pacientes y a su derecho a decidir libre e informadamente en el marco de la cultura propia.

California Press. Los Angeles California. 1998.

8. GReEN EC. The integration of Modern and Traditional Health Sectors in Swaziland. En: Anthropological Praxis Robert M, Wulff SJ, Fiske, eds. Wesview Press Boulder. London. 1997; 87-97.

9. Heiman CG. Culture, Health and Illness. Butterworth-Heinemann. Oxford. 1994.

10. BERLIN E, FowKes C. Teaching Framework for Cross-Cultural Health Care. Mayfield Publishing Company. California. 1998.

11. Heurtin-Roberts S, Reisin E. Health Beliefs and Compliance with Prescribed Medication for Hipertension Among Black Women. Boston: Reidel Publishing. 1992.

12. Harwood A. The Hot-Cold Theory of Disease: Implications for the Treatment of Puerto Rican Patients. Mayfield Publishing Company. California. 1998.

13. Alarcón A, Ancavil E, Cerda A, Hermosilia E, Maloueo V, Palma E. Una perspectiva cultural de la donación de órganos y tejidos. Ciencia y Enfermería 2001; 7: 67-75. 